\title{
Conclusion
}

\section{The Human Machine}

\section{at the Aboagora Symposium}

Aboagora 2013 discussed the complex relationships between man and machine, where not only may the human being itself be viewed as a corporeal machine, but it is also possible to interpret the machine as an extension of the human sensory system. After three days of lectures and workshops about the multifaceted relationship between man and the machine, the ontological dividing line between humans and machines was open to question. For example, while the human body can be defined as the ultimate machine - an assemblage of forces, actions and mechanisms ranging from the optics of the eye to the processes of cognition - the boundaries between man and machine may be blurred as technological devices are used as integral parts of the human body. Where do we draw the line between man and machine in such situations? The Aboagora symposium on 'The Human Machine' raised important questions about the ontological qualities and delineations of various entities.

We build machines, we program them and even try to give them the capacity to create, feel, think and decide by themselves. We use machines to improve our own faculties. They are integral parts of our functioning in daily life, work and entertainment. However machines are not merely things that we use; they are vehicles of human creativity. Machines are our collaborators in the creation of substance and the formulation of meaning. The definition of the 'machine', as it was scrutinized in several of the presentations in Aboagora 2013, went beyond the practical concept of a technological aid. The discussions which followed the lectures and workshops were lively and there were also some criticisms of the 'ontological blurriness' which has emerged in this 'mechanised' era.

As a conclusion the Aboagora symposium on 'The Human Machine' found that the assumed paradox between 'born humans' and 'built machines' is no longer a polarized one. Machines are our creations and as creators we want to give them a free will, the ability to be creative. Aboagora 2013 succeeded in problematising the simplistic division between man and the machine. In the future we should look more deeply into the souls of machines and humans.

In the year 2014 Aboagora's theme will be 'Chaos and Cosmos' (where cosmos is understood in its wider sense as 'order'). The symposium will be divided into three (day-long) sub-themes which are: 'Micro Cosmos', 'Macro Cosmos' and 'Chaos and Creation'. Aboagora will approach the fulfillment of this this seemingly limitless undertaking by looking at the latest developments in brain research, cosmology, physics, mathematics, big data, aesthetics, creativity, history, culture, religion and mythologies.

ANNA HAAPALAINEN

MA Anna Haapalainen is coordinator of the Aboagora symposium and doctoral candidate in comparative religion, University of Turku. Email: anna.haapalainen@utu.fi

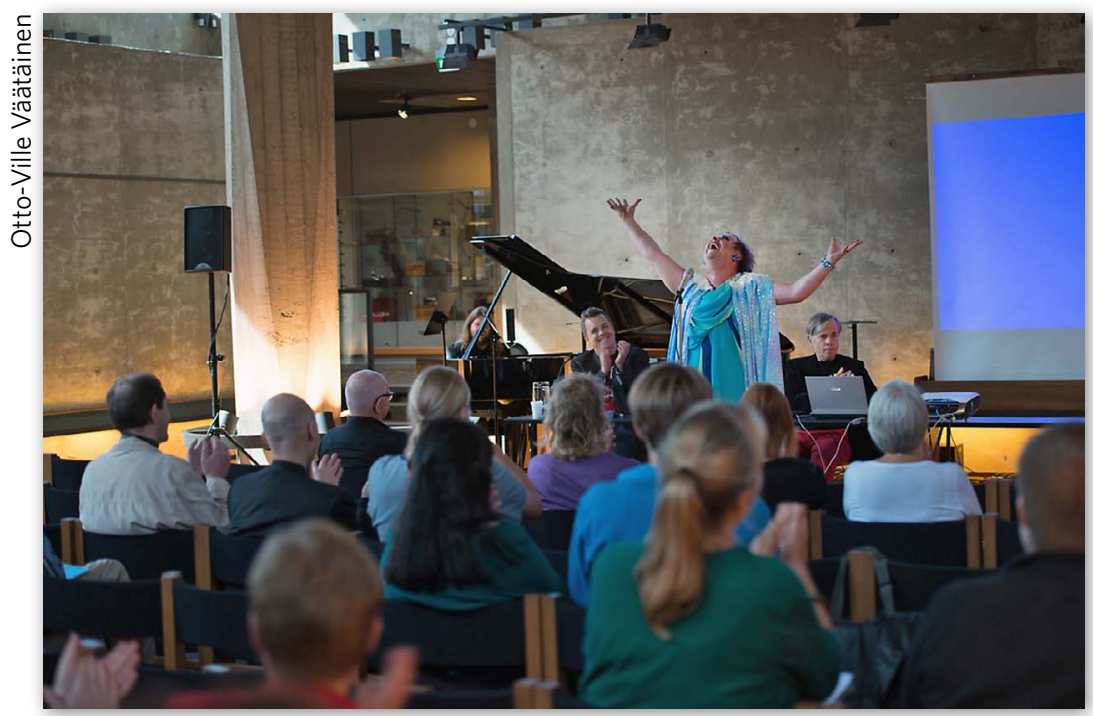

Drag queen Mega-Paula (Juha Rastas) performing at Aboagora 2013 during the session 'Technical Enchantments'. During the session, individual patterns of stress and recovery were discussed - a theme that Mega-Paula shared her thoughts and personal experience of. 